\title{
Is blockade of vascular endothelial growth factor beneficial for all types of diabetic retinopathy?
}

\author{
D. N. Sang • P. A. D'Amore
}

Received: 1 May 2008 / Accepted: 22 May 2008 /Published online: 8 July 2008

(C) Springer-Verlag 2008

Keywords Anti-VEGF. Bevacizumab - Diabetic macular oedema . Diabetic retinopathy Macular oedema . Neovascularisation $\cdot$ Pegaptanib $\cdot$ Proliferative diabetic retinopathy $\cdot$ Ranibizumab $\cdot$ VEGF

$\begin{array}{ll}\text { Abbreviations } \\ \text { AMD } & \text { age-related macular degeneration } \\ \text { DMO } & \text { diabetic macular oedema } \\ \text { ETDRS } & \text { Early Treatment Diabetic Retinopathy Study } \\ \text { PDR } & \text { proliferative diabetic retinopathy } \\ \text { READ } & \begin{array}{l}\text { Ranibizumab for Edema of the Macula } \\ \text { in Diabetes }\end{array} \\ \text { RPE } & \begin{array}{l}\text { retinal pigment epithelium } \\ \text { VEGF }\end{array} \\ & \text { vascular endothelial growth factor }\end{array}$

The pathophysiology of diabetic retinopathy leads to the clinical findings of increased retinal vascular permeability and progressive retinal ischaemia, and vascular endothelial

D. N. Sang $\cdot$ P. A. D'Amore $(\bowtie)$

Schepens Eye Research Institute,

20 Staniford Street,

Boston, MA 02114, USA

e-mail: patricia.damore@schepens.harvard.edu

D. N. Sang • P. A. D'Amore

Department of Ophthalmology, Harvard Medical School,

Boston, MA, USA

P. A. D'Amore

Pathology, Harvard Medical School,

Boston, MA, USA

D. N. Sang

Ophthalmic Consultants of Boston,

Boston, MA, USA growth factor (VEGF) has been identified as a key mediator in diabetic retinopathy. Loss of vision is most commonly a direct or indirect sequela of VEGF-mediated pathology, with ischaemia and/or oedema, retinal neovascularisation, vitreous haemorrhage and traction retinal detachment. Laser photocoagulation has been the standard of care in the treatment of both diabetic macular oedema (DMO) and proliferative diabetic retinopathy (PDR), and has been documented as reducing visual loss $[1,2]$. In the Early Treatment Diabetic Retinopathy Study (ETDRS), laser treatment for PDR reduced the incidence of severe vision loss by $50 \%$, and laser treatment for DMO reduced the incidence of moderate visual loss by $50 \%$ [2]. Although benefits were statistically significant, visual gains were limited.

Thus, there is clearly a need for better treatment outcomes. In this issue, Simó and Hernández review the current status regarding the widespread use of anti-VEGF drugs for the treatment of diabetic retinopathy [3]. AntiVEGF drugs are currently the mainstay of treatment for another neovascular ocular condition: the exudative form of age-related macular degeneration ('wet' AMD) involving neovascularisation arising from the subretinal or choroidal circulation under the macula, in the setting of pre-existent retinal pigment epithelial (RPE) degeneration (non-exudative 'dry' macular degeneration). In view of the known role of VEGF in the pathogenesis of wet AMD, and the successful outcomes associated with intravitreal anti-VEGF treatment for choroidal neovascularisation in wet AMD [4, 5], the same pharmacological therapeutic approaches are being embraced for both DMO and retinal neovascularisation in PDR. We question whether anti-VEGF will be as effective for the treatment of DMO as it is for either the treatment of retinal neovascularisation in PDR or choroidal neovascularisation in wet AMD. Since ischaemia-induced VEGF has 
been clearly implicated in the pathogenesis of retinal neovascularisation in PDR [6, 7], a favourable response of PDR to anti-VEGF therapies is not unexpected; a rapid response of retinal and iris neovascularisation has been reported following a single intravitreal anti-VEGF injection [8]. However, the response of DMO to anti-VEGF treatment appears to be much less robust. The role of VEGF in DMO is less clear. VEGF has been experimentally documented to induce retinal vessel permeability by altering the bloodretina barrier $[9,10]$. However, it is not evident that this is the predominant mechanism of vessel permeability in DMO.

\section{Anti-VEGF for DMO}

Intraocular injections of pegaptanib (selective $\mathrm{VEGF}_{165}$ blockade) and ranibizumab (pan-VEGF blockade) have been approved by the US Food and Drug Administration for the treatment of choroidal neovascularisation in wet AMD. Intraocular injection of bevacizumab (pan-VEGF blockade) has not been approved by the FDA but has been extensively and successfully employed as an off-label pan-VEGF antagonist in wet AMD. In contrast, with respect to DMO, pegaptanib is the only anti-VEGF agent to have shown favourable results, and has been investigated in a clinical trial that included 172 patients [11]. Studies on the use of ranibizumab and bevacizumab have not produced clear-cut results in DMO or have been short-term or subject to other limitations. The short-term Phase I Ranibizumab for Edema of the Macula in Diabetes (READ) pilot study of ten patients reported mean improvements of $85 \%$ for optical coherence tomography thickness and 12.3 ETDRS letters [12]. The Phase II READ-2 trial is currently underway. An additional recent open-label study that included ten patients also indicated a benefit [13]. Off-label bevacizumab has also appeared to offer a short-term benefit in some patients [14]. A modest response of DMO to bevacizumab was reported in a study of 51 patients with diffuse DMO, with no statistically significant increase in visual acuity and without normalisation of central macular thickness [15]. Interestingly, when outcomes of intravitreal bevacizumab treatment of DMO were compared with those of intravitreal cortisone (triamcinolone acetonide), better outcomes in terms of reduction of foveal thickness and visual results were found with triamcinolone [16]. Although the clinical benefit of intravitreal bevacizumab in DMO is not clear, it continues to be widely utilised.

\section{Permeability in AMD vs DMO}

Whereas vessel growth and leakage in wet AMD is highly associated with VEGF produced by 'activated' RPE cells and/or macrophages, VEGF has not been shown to be a major contributor to vascular permeability in DMO. Furthermore, cellular basis of the permeability in wet AMD is different from that in DMO. In wet AMD, new vessels arise from subretinal fenestrated choroidal (choriocapillaris) endothelium. These nascent vessels are abnormal in that they lack the typical pericyte association, have incomplete basement membranes and undergo continuous remodelling. In contrast, the presumed source of oedema in diabetes is the intraretinal vasculature. These vessels are the site of the blood-retinal barrier and are characterised by a high degree of pericyte coverage, extensive tight junctions, few pinocytotic vessels and a high transendothelial resistance. Although studies conducted to date suggest that a 'loosening' of the interendothelial junctions is likely to be a dominant factor in the increased permeability [17], the cause of the junctional disruption is not known. Thus, whereas the oedema in AMD is associated with VEGFinduced new vessel growth, the pathogenesis of DMO is less well understood. In addition, retinal pigment epithelium (RPE) permeability has been suggested to play a role in DMO [18]. Considering the distinct sources of permeability in the two pathologies and the distinct nature of two vasculatures, it is might be expected that the responses to anti-VEGF in AMD and DMO would not be the same.

\section{Possible side effects of anti-VEGF therapy}

Of concern are recent studies reporting the importance of VEGF in terms of both normal systemic vascular dependency $[19,20]$ and neuroprotection [21, 22]. In addition, the role of VEGF in the neuroprotection of retinal neuronal cells and RPE maintenance [14] raises issues concerning ocular safety, in addition to systemic safety, in an at-risk diabetic population. In light of the fact that continued antiVEGF treatment would be required to sustain any benefit from the treatment of ischaemia-related diabetic retinal dysfunction, consideration of its possible side effects is essential. None of the clinical trials performed to date have analysed the incidence of retinal ischaemia, retinal atrophy or thinning, or RPE degeneration to determine whether these are increased in response to anti-VEGF treatment.

Ocular effects Recent studies demonstrate the importance of VEGF in the adult and, most importantly for this discussion, specifically in the retina [23, 24]. VEGF is critical in the maintenance of fenestrated blood vessels such as the choriocapillaris, the glomerular capillaries and the choroid plexus [25-27]. In non-human primates, the intravitreal administration of bevacizumab has been demonstrated to reduce choriocapillaris fenestrations [27]. Endogenous VEGF was reported to play a role in the survival of retinal ganglion cells and was shown to be critical for the survival of retinal neuronal cells in response to ischaemic injury 
[23]. One study demonstrated that experimental systemic neutralisation of VEGF in mice leads to the death of a significant number of photoreceptors at 14 days, as measured by electroretinography, associated with a reduction in visual acuity [28]. These observations are consistent with the finding of mitochondrial damage to the inner segments of photoreceptors and increased apoptosis following intravitreal bevacizumab [29]. As the RPE is the major source of VEGF in the ocular fundus, we have hypothesised that RPE-derived VEGF is essential for the maintenance of the RPE-choriocapillary complex [30]. Of note, of the AMD patients treated for 12 months with ranibizumab in the Minimally Classic/Occult Trial of the Anti-VEGF Antibody Ranibizumab in the Treatment of Neovascular Age-Related Macular Degeneration (MARINA) and Anti-VEGF Antibody for the Treatment of Predominantly Classic Choroidal Neovascularization in Age-Related Macular Degeneration (ANCHOR) trials, $5.4 \%$ and $4.7 \%$, respectively, experienced a loss of visual acuity of $>15$ ETDRS letters, and this was primarily associated with increased RPE atrophy [31, 32]. A number of ongoing clinical trials are investigating the treatment of DMO with bevacizumab (Diabetic Retinopathy Clinical Research Network), ranibizumab (Wilmer Eye Institute at Johns Hopkins University, Baltimore, MD, USA) and VEGF-trap, a VEGF-neutralising fusion protein (Regeneron Pharmaceuticals, Tarrytown, NY, USA). The clinical risk of loss of normal vasculo- and neuroprotective VEGF function is not formally addressed in these studies.

Systemic effects The risk and degree of systemic exposure from intravitreal anti-VEGF injections are not known, although circulating anti-VEGF antibodies have been measured following intravitreal administration [33]. Clinically, there are many anecdotal reports of benefit to the fellow eye following injection of the first eye with an anti-VEGF agent, suggesting systemic exposure, but clinical trials of anti-VEGF drugs in the treatment of wet AMD and diabetic retinopathy have not been adequately powered to address the incidence of systemic complications. The most common risks of systemic anti-VEGF include thromboembolic events, hypertension and proteinuria [34]. As pointed out by Simó and Hernández in their review, there is a greater concern even with low dose systemic exposure in the diabetic population with their baseline-increased risk of cardiovascular and peripheral small vessel disease [3].

\section{Conclusion}

Wet AMD, DMO and neovascularisation in PDR arise from very different pathogenic mechanisms. In light of the great success of anti-VEGF agents in the treatment of wet AMD, there have been high expectations for the treatment of all subtypes of diabetic retinopathy by the intravitreal injection of these agents. However, the response to treatment of DMO by VEGF blockade is not prolonged and is subject to significant variability. This is in distinct contrast to the rapid response of both iris and retinal neovascularisation in PDR and of choroidal neovascularisation in wet AMD. The role of selective blockade of $\mathrm{VEGF}_{164}$ (pegaptanib) vs panVEGF blockade (ranibizumab, bevacizumab) remains to be clarified. DMO without a significant ischaemic component may not benefit from pan anti-VEGF therapy as much as pre-proliferative or proliferative diabetic retinopathy with marked ischaemia. The use of anti-VEGF therapy as an adjunct to traditional laser treatment is being investigated (Diabetic Retinopathy Clinical Research Network; http:// www.drcr.net/).

Clearly, VEGF is not the sole pathogenic mechanism underlying the different forms of diabetic retinopathy; there are major challenges and influences from systemic factors, including glycaemic control, hypertension, lipid metabolism, inflammatory and immunological factors and genetic factors such as polymorphisms in the gene encoding VEGF $[35,36]$. Nonetheless, VEGF appears to play a critical role in the pathophysiology of diabetic retinopathy associated with retinal ischaemia. Anti-VEGF blockade for PDR offers a major benefit. However, the extent to which VEGF blockade is beneficial for DMO is currently being investigated in prospective clinical trials and is not clear-cut from pilot studies. For these reasons, a heightened awareness of the potential systemic risks and risks of anti-angiogenesis in the diabetic patient population should be exercised when prescribing treatment with anti-VEGF antagonists, and every attempt should be made to minimise the risks. The use of anti-VEGF agents in combination with laser treatment and/or other anti-permeability drugs may provide opportunities for maximising the benefits of anti-VEGF therapy while minimising both systemic and ocular risk.

Duality of interest DNS has served on advisory boards for Novartis, QLT and OSI-Eyetech, and received research funds from QLT.

\section{References}

1. Kinyoun J, Barton F, Fisher M, Hubbard L, Aiello L, Ferris F 3rd (1989) Detection of diabetic macular edema. Ophthalmoscopy versus photography-Early Treatment Diabetic Retinopathy Study report number 5. The ETDRS Research Group. Ophthalmology 96:746-750

2. Early Treatment Diabetic Retinopathy Study Research Group (1991) Early Treatment Diabetic Retinopathy Study design and baseline patient characteristics. ETDRS report number 7. Ophthalmology 98:741-756 
3. Simó R, Hernández C (2008) Intravitreous anti-VEGF for diabetic retinopathy: hopes and fears for a new therapeutic strategy. Diabetologia doi 10.1007/s00125-008-0989-9

4. Rosenfeld PJ, Brown DM, Heier JS et al (2006) Ranibizumab for neovascular age-related macular degeneration. $\mathrm{N}$ Engl J Med 355:1419-1431

5. Brown DM, Regillo CD (2007) Anti-VEGF agents in the treatment of neovascular age-related macular degeneration: applying clinical trial results to the treatment of everyday patients. Am J Ophthalmol 144:627-637

6. Miller JW, Adamis AP, Shima DT et al (1994) Vascular permeability factor/vascular endothelial cell growth factor is temporally and spatially correlated with ocular angiogenesis in a primate model. Am J Pathol 145:574-584

7. Aiello LP, Avery RL, Arrigg PG et al (1994) Vascular endothelial growth factor in ocular fluid of patients with diabetic retinopathy and other ocular disorders. N Engl J Med 331:1480-1487

8. Avery RL (2006) Regression of retinal and iris neovascularization after intravitreal bevacizumab (Avastin) treatment. Retina 26:352-354

9. Tolentino MJ, Miller JW, Gragoudas ES, Chatzistefanou K, Ferrara N, Adamis AP (1996) Vascular endothelial growth factor is sufficient to produce iris neovascularization and neovascular glaucoma in a nonhuman primate. Arch Ophthalmol 114:964970

10. Aiello LP, Bursell SE, Clermont A et al (1997) Vascular endothelial growth factor-induced retinal permeability is mediated by protein kinase $\mathrm{C}$ in vivo and suppressed by an orally effective beta-isoform-selective inhibitor. Diabetes 46:1473-1480

11. Cunningham ET Jr, Adamis AP, Altaweel M et al (2005) A Phase II randomized double-masked trial of pegaptanib, an anti-vascular endothelial growth factor aptamer, for diabetic macular edema. Ophthalmology 112:1747-1757

12. Nguyen QD, Tatlipinar S, Shah SM et al (2006) Vascular endothelial growth factor is a critical stimulus for diabetic macular edema. Am J Ophthalmol 142:961-969

13. Chun DW, Heier JS, Topping TM, Duker JS, Bankert JM (2006) A pilot study of multiple intravitreal injections of ranibizumab in patients with center-involving clinically significant diabetic macular edema. Ophthalmology 113:1706-1712

14. Arevalo JF, Fromow-Guerra J, Quiroz-Mercado H et al (2007) Primary intravitreal bevacizumab (Avastin) for diabetic macular edema: results from the Pan-American Collaborative Retina Study Group at 6-month follow-up. Ophthalmology 114:743-750

15. Haritoglou C, Kook D, Neubauer A et al (2006) Intravitreal bevacizumab (Avastin) therapy for persistent diffuse diabetic macular edema. Retina 26:999-1005

16. Shimura M, Nakazawa T, Yasuda K et al (2008) Comparative therapy evaluation of intravitreal bevacizumab and triamcinolone acetonide on persistent diffuse diabetic macular edema. Am J Ophthalmol 145:854-861

17. Weis SM, Cheresh DA (2005) Pathophysiological consequences of VEGF-induced vascular permeability. Nature 437:497-504

18. Schneck ME, Shupenko L, Adams AJ (2008) The fast oscillation of the EOG in diabetes with and without mild retinopathy. Doc Ophthalmol 116:231-236
19. Maharaj AS, Saint-Geniez M, Maldonado AE, D'Amore PA (2006) Vascular endothelial growth factor localization in the adult. Am J Pathol 168:639-648

20. D'Amore PA (2007) Vascular endothelial cell growth factor-A: not just for endothelial cells anymore. Am J Pathol 171:14-18

21. Zachary I (2005) Neuroprotective role of vascular endothelial growth factor: signalling mechanisms, biological function, and therapeutic potential. Neurosignals 14:207-221

22. Kilic U, Kilic E, Jarve A et al (2006) Human vascular endothelial growth factor protects axotomized retinal ganglion cells in vivo by activating ERK-1/2 and Akt pathways. J Neurosci 26:12439-12446

23. Nishijima K, Ng Y-S, Zhong L et al (2007) VEGF-A is a survival factor for retinal neurons and a critical neuroprotectant during the adaptive response to ischemic injury. Am J Path 171:53-67

24. Saint-Geniez M, D'Amore PA (2004) Development and pathology of the hyaloid, choroidal and retinal vasculature. Int J Dev Biol 48:1045-1058

25. Kamba T, Tam BY, Hashizume H et al (2006) VEGF-dependent plasticity of fenestrated capillaries in the normal adult microvasculature. Am J Physiol Heart Circ Physiol 290:H560-H576

26. Maharaj AS, Walshe TE, Saint-Geniez M et al (2008) VEGF and TGF-beta are required for the maintenance of the choroid plexus and ependyma. J Exp Med 205:491-501

27. Peters S, Heiduschka P, Julien S et al (2007) Ultrastructural findings in the primate eye after intravitreal injection of bevacizumab. Am J Ophthalmol 143:995-1002

28. Maharaj AS, Saint-Geniez M, Walshe TE et al (2008) VEGF is required for neuroretina survival and function. Invest Ophthalmol Vis Sci 49:E-5836 (Abstract)

29. Inan UU, Avci B, Kusbeci T, Kaderli B, Avci R, Temel SG (2007) Preclinical safety evaluation of intravitreal injection of full-length humanized vascular endothelial growth factor antibody in rabbit eyes. Invest Ophthalmol Vis Sci 48:1773-1781

30. Saint-Geniez M, Maldonado AE, D'Amore PA (2006) VEGF expression and receptor activation in the choroid during development and in the adult. Invest Ophthalmol Vis Sci 47:3135-3142

31. Rosenfeld PJ, Brown DM, Heier JS et al (2006) Ranibizumab for neovascular age-related macular degeneration. N Engl J Med 355:1419-1431

32. Brown DM, Kaiser PK, Michels M et al (2006) Ranibizumab versus verteporfin for neovascular age-related macular degeneration. N Engl J Med 355:1432-1444

33. van Wijngaarden P, Coster DJ, Williams KA (2005) Inhibitors of ocular neovascularization: promises and potential problems. JAMA 293:1509-1513

34. Hurwitz H, Fehrenbacher L, Novotny W et al (2004) Bevacizumab plus irinotecan, fluorouracil, and leucovorin for metastatic colorectal cancer. N Engl J Med 350:2335-2342

35. Yang B, Cross DF, Ollerenshaw M, Millward BA, Demaine AG (2003) Polymorphisms of the vascular endothelial growth factor and susceptibility to diabetic microvascular complications in patients with type 1 diabetes mellitus. J Diabetes Complications 17:1-6

36. Buraczynska M, Ksiazek P, Baranowicz-Gaszczyk I, Jozwiak L (2007) Association of the VEGF gene polymorphism with diabetic retinopathy in type 2 diabetes patients. Nephrol Dial Transplant 22:827-832 